\title{
The Role of Pd-L1 on Tumor Cells in Host Immune System Escape and Tumor Immunotherapy by Pd-L1 Blockade; Immune Checkpoint Blockade in Cancer Immunotherapy
}

\section{Mehmet Eraslan*}

Çanakkale Onsekiz Mart University, Faculty of Science and Literature, Turkey

*Corresponding Author: Mehmet Eraslan, Çanakkale Onsekiz Mart University, Faculty of Science and Literature, Turkey.
Received: August 26, 2021

Published: September 15, 2021

(C) All rights are reserved by Mehmet

Eraslan.

\section{Abstract}

Immune checkpoints are a group of inhibitory mechanisms that are encoded into the immune system. Mice treated with antiPD L1 or PD-1-deficient animals showed increased tumorigenesis and invasiveness in syngeneic hosts when compared to parental tumor cells lacking PD-L. The classical type of programmed cell death is distinguished by its morphological hallmarks of apoptosis and its reliance on de novo RNA and protein production. The PD-1 gene was active in both stimulated 2B4.11 and IL-3-depleted LyD9 cells. The findings imply that the genetic landscape of lung malignancies influences anti-PD-1 therapeutic responsiveness. Immune resistance can be overcome by blocking the inhibitory receptor programmed death 1 (PD-1), which is produced by T cells. An antibody that precisely targets PD-1, was considered for anticancer efficacy and safety. There is a link between PD-L1 expression on tumor cells and objective response. In patients with advanced malignancies such as non-small cell lung cancer, melanoma, and renalcell cancer, antibody-mediated PD-L1 inhibition resulted in long-term tumor shrinkage and disease stability.

Keyword: Immunotherapy; PD-1 and PD-L1; Anti-PD-L1; Cancer; Blockade of CTLA-4; Tumor

\section{Introduction}

Immune checkpoint blockade appears to unlock the antitumor immune response's potential in a way that is altering human cancer therapies. T cells have been the main focus of efforts to alter anti-tumor immunity therapeutically. The principal drugs in present clinical trials are agonists of co-stimulatory receptors or antagonists of inhibitory signal. Antibodies that block immune checkpoints do not target tumor cells directly, instead they target lymphocyte receptors or their ligands. The two immunological checkpoints primarily investigated in the context of cancer immunotherapy are CTLA4 and PD1. Antibodies that inhibit one or both of these receptors suggest that antitumor immunity can be improved. Researchers have discovered that inhibitors for several of these checkpoints are in the works [1-4].
CD28 and ICOS are positive costimulatory receptors that bind with the ligands of the B7 family on professional antigen-presenting cells and are required for antigen-specific $\mathrm{T}$ cell activation and proliferation. Moreover, the cytotoxic T lymphocyte (CTL)-A-4 and -PD-1 receptors on T cells have opposing roles. However, PD-1, interacts with two different ligands, PD-L1 and PD-L2, that are found on antigen-presenting cells as well as other cell types in nonlymphoid tissues $[2,5,6]$. Based on increasingly sophisticated assay techniques, there is growing evidence that a particular $\mathrm{T}$ cell immune response may be produced against a variety of cancers in humans and mice. It is also been shown that unchanged self-antigens generated on tumor cells in an aberrant or tissue-specific manner might function as important tumor rejection antigens, implying that antitumor immunity is partly an autoreactive response $[1,5,7,8]$. 
The blockade of CTLA-4 was indicated to augment the specific tumor immunity and to induce significant inhibition of tumors in vivo in a number of experimental murine models. In this report was explored the possibility for involvement of PD-1/PD-L1 system in tumor immunity by using experimental tumor models. The presence of PD-L1 on fundamentally immunogenic tumor cells confers a powerful evasion mechanism from host $\mathrm{T}$ cell immunity, raising the prospect that blocking the PD-1-PD-L interaction could be a useful method for targeted tumor immunotherapy $[2,5,6,8]$.

\section{CTLA4 biology}

The immune-checkpoint receptor CTLA4 was the first to be therapeutically targeted. It inhibits the function of CD28, a T-cell co-stimulatory receptor. The exact mechanism of action is unknown, but it is assumed to work by increasing the activity of effector CD4+ T cells and inhibiting TReg cell-dependent immunosuppression. It's been suggested that its presence on the surface of T cells inhibits $\mathrm{T}$ cell activation $[2,9,10]$.

The lengthy journey from mice to FDA approval for CTLA4blocking antibodies in clinical trials

Anti-tremelicumab in melanoma was the first randomized Phase III clinical study to be completed. Ipilimumab-treated individuals had a survival rate of $18 \%$ after two years. The discovery of continued responses and survival after a very short course of therapy lends credence to the idea that immune-based treatments can re-educate the immune system to keep tumors in control after the therapeutic intervention is over. The proportion of long-term survival was higher than the percent of objective responders in this and other investigations, authors say $[2,5,9]$.

\section{Blocking the PD1 signaling pathway}

PD1, is emerging as a prospective target, highlighting the wide range of molecularly defined immunological alterations capable of generating antitumor immune responses by the patient's own immune system $[2,5,9]$.

\section{The PD1 pathway's biology}

Unlike CTLA4, PD1's main function is to suppress T cell activation in peripheral tissues. In the tumor microenvironment, this corresponds to a significant immune resistance mechanism. PDL1 (PDL1) and PDL2 (PDL2) are the two ligands for PD1 (B7-DC and CD273) These members of the B7 family have a 37 percent se- quence homology and were created via gene duplication. Understanding the relevance of these varied interactions in different cancer contexts is critical for the selection of antibodies and recombinant ligands for clinical usage. This article has been amended to make it clear that the interaction between PD1 and the ligand PDL1 was initially meant to be highlighted [3,4,9-15].

Constitutive versus adaptive immune resistance in the regulation of PD1 and its ligands in tumors

A substantial proportion of tumor-infiltrating lymphocytes (TILs) from a variety of tumor types express PD1. Increased PD1 expression on CD8+ TILs might indicate a fatigued or anergic condition. PDL1 expression in mouse tumor cells is forced, which decreases local antitumour $\mathrm{T}$ cell responses. This set of discoveries lays the groundwork for inhibiting the PD1 pathway to improve anti-tumor effector activities in the tumor microenvironment. Furthermore, PD1 suppresses lymphocyte activity only when its ligands, PDL1 and PDL2, bind to it $[3,8,10,16,17]$.

\section{Evidence of PD1 blockade's clinical efficacy}

The initial clinical trial's results indicated that the effects are long-lasting. All objective responders in the original Phase I study remained in remission for more than a year after treatment ended. In renal cancer, similar response rates have been found. In the original study with anti-PD1, just one patient out of 39 suffered a serious immune-related side event. Anti-PD1 is now being studied in lung cancer. Anti-PD1 therapy appears to cause less immunerelated side effects than anti-CTLA4 therapy. PDL1. expression and response were shown to be strongly linked in analyses of 9 patients who were treated with anti-PSD1 in the first Phase One study. Antibodies targeting PD1 or PDL 1 are being developed and tested by a number of firms $[9,18]$.

Several immunological checkpoints that appear to be promising

T helper 1 (TH1) cell responses are inhibited by TIM3, whose ligand is galectin 9. Antibodies to TIM3 improve antitumor immunity. The increased $\mathrm{T}$ cell responses observed in Btla-knockout mice led to the discovery of BTLA as an inhibitory receptor on $\mathrm{T}$ cells. A TNF family member interacting with an immunoglobulin supergene family member is an uncommon occurrence. The main challenge is which of the more than 20 receptors should be used as a therapeutic target. Tumors have a high rate of cell death due 
to cell turnover, and dying cells emit adenosine. In mice with A2a R-knocking out animals, tumour vaccinations are substantially more effective against existing tumors. Killer inhibitory receptors were first identified as critical regulators of NK cell killing capacity. Their suppressive effect on T cells and APCs has received little attention. If not properly engaged, NK cells can deliver significant anti-tumour action [9,15,19-21].

The majority of cells that undergo programmed death require de novo RNA and protein synthesis. Inhibitors of macromolecular synthesis, such as actinomycin D or cycloheximide, can prevent cell death. These findings show that planned cell death requires the expression of at least a few genes, if not particular genes. Apoptosis causes chromatin to condense at the nucleus' periphery, but mitochondria and other organelles remain unharmed. The fragmentation of DNA into oligonucleosomal particles is a distinctive biochemical property of apoptotic cells. Apoptosis is frequently connected with programmed cell death, yet some cells experiencing programmed death may not appear to be experiencing apoptosis. Furthermore, apoptotic cell death can be caused even when no macromolecular synthesis is present. It's vital to remember that apoptosis isn't the same as programmed cell death $[2,3,7,13,22]$.

The death of self-reactive immature $\mathrm{T}$ cells is a well-known example of this type. To extract such genes, an experimental environment must be established in which the traditional form of programmed cell death may be easily generated. Four mouse cell lines, shown below, that can die by apoptosis in response to stimulation are ideal candidates for use in this study. A T-cell hybridoma 2B4. 11 dies when activated with its corresponding antigen or a combination of ionomycin and phorbol 12-myristate 13-acetate, just like immature $\mathrm{T}$ cells. When anti-IgM antibodies crosslink the surface IgM molecules of an immature B-cell line, WEHI-231, the cells perish. LyD9, a lymphoid/myeloid progenitor cell line, and CTLL-2, a cytotoxic T-cell line, both require interleukin-3 (IL-3) and interleukin-2 (IL-2) for survival and proliferation, and both perish when the growth factors are removed from the culture. PD-1, a novel member of the immunoglobulin gene class, was identified and described as a programmed cell deathspecific cDNA. The expression of the PD-1 mRNA was confined to the thymus and increased when the TCR -CD3 complex on thymocytes was disrupted, causing cell death in the thymus $[2,3,7,11,13,21,22]$.

Many genetic and epigenetic changes occur in human malignancies, resulting in neoantigens that the immune system may recognize. Tumors acquire resistance mechanisms such as local immune suppression, tolerance induction, and systemic T-cell signaling failure. Inhibiting the link between PD-1 and PD-L1 can improve T-cell responses in vitro and mediate anticancer activity in preclinical models. The anti-PD-1 monoclonal antibody BMS-936558 was given as a single dose to 39 patients with advanced solid tumors in a dosage-escalation trial. This pilot research revealed a positive safety profile and tentative indication of therapeutic effectiveness, laying the groundwork for the present multi-dose experiment enrolling patients with various malignancies. In this study, the clinical outcomes of 296 individuals [9,21,23,24].

\section{Discussion and Conclusion}

\section{Baseline Patient Characteristics and Treatment}

Between October 2008 and February 24, 2012, 296 patients with advanced solid tumors started receiving anti-PD-1 antibody therapy. The bulk of the patients had been extensively pretreated, with $47 \%$ having had at least three previous regimens. Immunotherapy and BRAF inhibitors in melanoma patients, as well as platinum-based chemotherapy in lung cancer patients, were notable past treatment. The overall treated population had identical baseline characteristics to the efficacy population $[7,16,24]$.

Patients in both the safety and efficacy populations had fairly comparable baseline demographic characteristics. 86 percent of treated patients had previously undergone chemotherapy, and $28 \%$ had previously had immunologic or biologic treatment. Immunotherapy (56 percent) and BRAF inhibitors (9\%) were previously used in individuals with melanoma, according to tumor type $[6,7,16,24]$.

\section{Safety}

62 patients (21\%) had died as of the date of analysis, with illness progression being the most prevalent cause of death. Fatigue, rash, diarrhea, pruritus, reduced appetite, and nausea were all common treatment-related side effects. In 32 of the 296 individuals, drugrelated major adverse events occurred (11 percent) Pneumonitis was the cause of three drug-related fatalities (1 percent): two in patients with non-small-cell lung cancer and one in a patient with colorectal cancer. Given the limited number of patients and varied results, the efficacy of immunosuppression was unknown, as was the amount of dosages administered. Across all dosage levels evaluated, the variety, frequency, and severity of treatment-related side events were generally consistent. Returning to the original article $[14,18,20,24]$.

86 percent of the patients who were treated had already had chemotherapy, and the maximum tolerable dosage was not achieved. Rashes, hypothyroidism, hepatitis, and sarcoidosis were among the drug-related side effects of particular relevance. In 11 of the 207 patients, there were serious adverse events that investigators thought were attributable to the medication. 45 individuals $(22 \%)$ had died by the time the data was shut off. Disease progression was the leading cause of mortality $[14,15,18,20]$ 
The Role of Pd-L1 on Tumor Cells in Host Immune System Escape and Tumor Immunotherapy by Pd-L1 Blockade; Immune Checkpoint Blockade in Cancer Immunotherapy

\section{Clinical activity}

At all dosages examined, antitumor activity was demonstrated. A significant number of patients with non-small-cell lung cancer, melanoma, or renal-cell carcinoma, as well as diverse locations of metastases, showed objective responses. All 14 patients who had objective responses had been on therapy for at least 24 weeks prior to data analysis, and eight of them had a response that lasted at least 24 weeks. Five of the 14 patients had objective responses that lasted a year or more, while two of the 14 patients had responses that lasted less than a year. Five patients (7 percent) with lung cancer, all of whom had nonsquamous tumors, had stable illness for 24 weeks. Patients with colorectal or prostate cancer, as well as any non-small-cell histologic type, had no objective responses. At dosages ranging from 0.1 to $10.0 \mathrm{mg}$ per kilogram, 26 objective responses in melanoma patients were reported. Response rates per dosage level ranged from 19 to $41 \%$. 7 of 17 individuals had objective responses at a dosage of $3.0 \mathrm{mg}$ per kilogram (41\%t) Objectives were achieved in 4 of 17 (24\%) patients with renal-cell cancer treated with doses of $1.0 \mathrm{~g} / \mathrm{kg}$ and 5 of $16(31 \%)$ patients treated with doses of $10 \mathrm{~g} / \mathrm{kg}[12,16,25]$.

At dosages of $1 \mathrm{mg}$ per kilogram or greater, clinical action was found. Melanoma, non- small-cell lung cancer, renal-cell carcinoma, and ovarian cancer patients all showed objective responses. In four more individuals, the number of target lesions decreased over time despite the existence of new lesions. Patients who had undergone a variety of procedures showed antitumor responses or had longterm stable illness $[12,16,25]$.

\section{Pharmacokinetics and pharmacodynamics}

The median time from the commencement of infusion to the maximal concentration of anti-PD- 1 antibody was 1 to 4 hours. The antibody's pharmacokinetics were linear, with peak concentration and area under the curve increasing in a dose-proportional manner. In PBMCs from 65 melanoma patients who received a single cycle of anti-PD-1 antibody at a dosage of 0.1 to $10.0 \mathrm{mg}$ per kilogram every two weeks [22,23].

In 131 individuals studied, serum levels of anti-PD-L1 antibody rose in a dosedependent manner from 1 to $10 \mathrm{mg}$ per kilogram. Anti-PD-L1 antibody has a half-life of around 15 days, according on population pharmacokinetics. At the end of one therapy cycle, the occupancy of the PD-L 1 receptor on CD3+ peripheral blood mononuclear cells was measured in 29 patients with melanoma $[15,22,23]$.

\section{PD-L1 expression in tumors}

PD-L1 expression on the surface of tumor cells was examined in 61 pre-treatment tumor tissues from 42 individuals. Immunohistochemical examination of biopsy tissues from 25 of the 42 patients revealed PD-L1 expression. Nine (36\%) of the 25 patients achieved an objective response. There was no objective response in any of the 17 patients with PD L1- negative tumors. This analysis is based on optional biopsies in a non-random subset of the population, and testing of a statistical hypothesis was not prespecified. Optional biopsies in a non-random subset of the population were used in this investigation, and no statistical hypothesis was tested $[15,20]$.

Characterization of apoptotic deaths of 2B4. 11, WEHI-231, LyD9 and CTLL-2

Actinomycin D (70-200ng/ml) was not toxic to 2B4. 11 and LyD9, but was highly toxic to both WEHI-231 and CTLL-2. As little as 2-3ng of actinomycin D induced apoptotic deaths in the two cell lines without any other stimulation. Researchers also looked at 5,6- dichloro1,B-D-ribofuranosylbenzimidazole (DRB), a less hazardous transcriptional inhibitor. 2B4. When 11 cells were stimulated with a mixture of ionomycin and PMA for 6 hours, the DNA fragmentation associated with apoptotic cell death was visible. The addition of actinomycin D at the time of stimulation virtually totally reduced this fragmentation. This clearly shows that de novo RNA synthesis is essential for 2B4 death mediated by stimulation. Actinomycin D supplied 2 hours after stimulation only marginally suppressed fragmentation, and no suppression was shown when actinomycin D was administered 3, 4, or 5 hours after stimulation $[21,26]$.

When compared to the death of $2 \mathrm{~B} 4$, the RNA synthesis required for the death of LyD9 continues for a longer amount of time. Cycloheximide, on the other hand, may successfully inhibit DNA fragmentation in activated WEHI-231 cells. These findings imply that anti-IgM antibody-mediated death of WEHI-23 1 requires de novo protein synthesis but not RNA production [21].

Isolation of cDNA clones for mRNAs induced upon programmed cell death

Even under the most restrictive conditions, the restriction maps of the four clones overlapped, and the four clones hybridized to one another. PD-1 was the name of the gene from which the four cDNAs were generated. A conventional cDNA library of stimulated 2B4. 11 mRNA yielded another clone with a longer insert (PD-l cDNA \# 7) $[9,19]$. 
The Role of Pd-L1 on Tumor Cells in Host Immune System Escape and Tumor Immunotherapy by Pd-L1 Blockade; Immune Checkpoint Blockade in Cancer Immunotherapy

\section{Structure of PD- 1 cDNA}

The extracellular domain of the PD-1 protein is similar to some members of the immunoglobulin superfamily, according to a comparison of its amino acid sequence with all other sequences in the database. The projected PD-1 protein's cytoplasmic domain comprises a variant form of the consensus sequence present in the cytoplasmic tails of most antigen receptor and Fc receptor polypeptides $[9,13,16]$.

\section{Cell death-associated expression of PD-1 mRNA}

Researcher found a small amount of PD-l mRNA expression in unstimulated 2B4.11 cells, however it was greatly increased in stimulated 2B4.11 cells. The expression of PD1 mRNA in stimulated 2B4. II cells peaked at $-3 \mathrm{~h}$ after stimulation and then declined somewhat at $5 \mathrm{~h}$. The WR19L mouse T-cell lymphoma line, whose apoptotic death may be caused by tumor necrosis factor-a within 2-3 hours of incubation, was discovered to express PD-I mRNA, but the expression was not increased by TNF-a treatment. The TNFa-sensitive L929 murine cell line did not express PD-I mRNA. Researchers found substantial DNA breakage in the thymus of mice injected with anti-CD3 mAb, as well as increased PD-I mRNA expression at 8- and 12-hours following injection. In the thymus of phosphate-buffered saline (PBS)-injected mice, however, neither DNA fragmentation nor an increase in PD-I expression were found. Researchers found substantial DNA breakage in the thymus of mice injected with anti-CD3 $\mathrm{mAb}$, as well as a 3-fold increase in PD-I mRNA expression at 8- and 12-hours following injection $[8,21,26]$.

Anti-PD-1 therapeutic response may be influenced by the mutational landscape of NSCLCs. Patients treated with pembrolizumab, a humanized immunoglobulin G (IgG) 4-kappa isotype antibody to PD-1, had their exomes sequenced. Clinical effectiveness was linked to a higher somatic nonsynonymous mutation load. Researchers discovered harmful mutations in a number of genes involved in DNA repair and replication. Both PRKDC, the catalytic component of DNA-dependent protein kinase (DNA-PK), and RAD17, which are necessary for normal DNA replication, have nonsense mutations. Using the methodology researchers previously discussed, researchers looked at the landscape of neoantigens. Researchers next examined all 34 exomes collectively to determine how patterns of mutational changes were associated with clinical benefit to pembrolizumab. Efficacy was greatest in patients with tumors harboring the smoking signature. C-to-A transversions were more frequent, and C-To-T transitions were less frequent, in DCB compared to NDB. KRAS mutations were discovered in 7 of 14 tumors from DCB patients compared to 1 of 17 in the NDB group, a find- ing that could be explained by the link between smoking and KRAS mutations in NSCLC. There were no mutations or copy-number alterations in antigen-presentation pathway-related genes or CD274 that were linked to responsiveness or resistance $[16,19,21]$.

$\mathrm{T}$ cell recognition of cancers relies upon presentation of tumor specific antigens on MHC molecules. Anti-PD-1-induced neoantigen-specific $\mathrm{T}$ cell reactivity can be observed within the peripheral blood compartment. The finding that nonsynonymous mutation burden more closely associates with pembrolizumab clinical benefit than total exonic mutation burden suggests the importance of neoantigens in dictating response to anti- PD-1 therapy. Researchers believe that the findings have an important impact on their understanding of response to Anti- PD L1 therapy and on the application of these agents in the clinic. A monoclonal antibody that blocked the immune suppressive ligand PD-L1 resulted in longterm tumor regression. Patients with non-small-cell lung cancer, melanoma, renal-cell cancer, and ovarian cancer who have metastasized 7,20. At dosages up to $10 \mathrm{mg}$ per kilogram, 9 percent of patients experienced grade 3 or 4 adverse events that were thought to be drug-related. Anti-PD-L1 antibody infusion responses were detected; however, they were modest in most individuals. The capacity of anti- PD-L 1 treatment to maintain response across many tumor types was a key characteristic. Given the advanced state of the disease and earlier therapies, this was quite noteworthy. Because $\mathrm{T}$ cells in the peripheral blood express PD- L1, in vitro receptor occupancy may be assessed. In early studies, the frequency of objective responses for anti-PD-L1 antibody appeared to be slightly lower than for anti-PD-1 antibody. Additional research is needed to establish which individuals are likely to respond and the optimum therapeutic dosage. These findings establish the PD-1-PD-L 1 pathway as a critical target for therapeutic intervention in cancer $[8,16,17,19,23]$.

On activation with the specified target, P815 tumor cells, the $2 \mathrm{C}$ CTL clone exhibited substantial PD-1 (Figure 1A). Because neither PD-L1 (Figure 1B) nor PD-L2 were expressed in P815 cells, three separate P815 transfectants stably expressing PD-L1 were created. ${ }^{4}$ In the culture, all of the P815/PD-L1 clones exhibited the same level of H-2Ld antigen and proliferated at the same rate as the parental cells. The P815/PD-L1 clones, on the other hand, were less vulnerable to the cytotoxic action of 2C CTL than the parental P815 cells. The lower susceptibility of the P815/PD-L1 clone to 2C CTL was almost fully reversed when anti-PD-L1 mAb F(ab')2 was added to the test culture, demonstrating that it was not attributable to a clonespecific characteristic. The findings indicate that the interac- 
The Role of Pd-L1 on Tumor Cells in Host Immune System Escape and Tumor Immunotherapy by Pd-L1 Blockade; Immune Checkpoint Blockade in Cancer Immunotherapy

tion of CTL PD-1 with PDL1 on particular target cells inhibited T cell receptor-mediated cytotoxic activity. Inhibition of carcinogenesis in endogenously expressed PD-L1 myeloma cells in normal syngeneic mice treated with anti-PD L1 Ab or PD-1-deficient animals. ${ }^{2,4,8,16}$

These findings suggest that by suppressing the effects of negative immunoreceptors, weak immune responses against tumors can be transformed into more robust ones that are effective for tumor suppression and rejection. PD-L1 expression on tumor cells, on the other hand, protects them from direct immune attack by specific CTL at the effector level, at least in vitro, and increases tumorigenicity in vivo, according to the current findings. In any event, present results suggest strongly that effective blockade of PD-1-PD-L interaction in vivo should provide a promising strategy of immunotherapy for selected tumors expressing PD-L. It would also be interesting to examine whether there are any synergistic therapeutic effects between blockade of PD-1 and CTLA- 4 on the tumors in vivo $[1,2,8,9,11,13,21]$

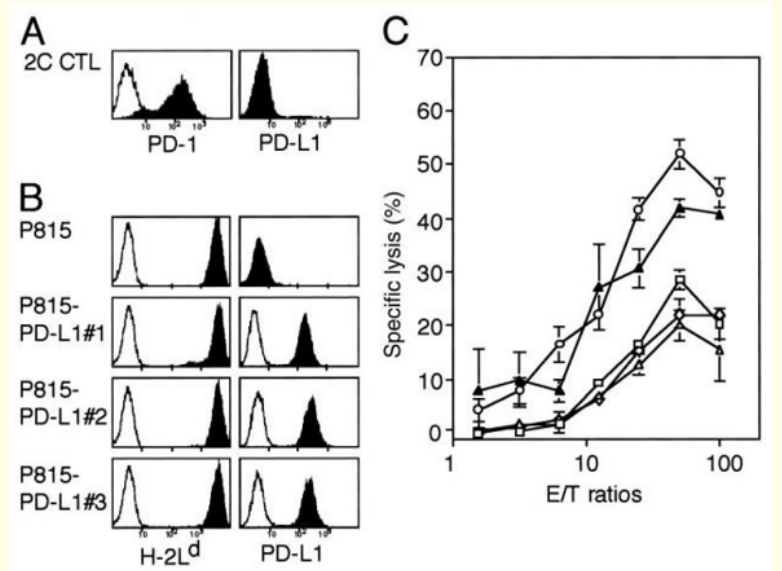

Figure 1: Engagement of the PD-1 receptor with PD-L1 on particular target cells inhibits the cytotoxic activity of the CTL clone. (Both A and B) Flow cytometry was used to examine the expression of PD-1, H-2Ld, and PD-L1 on 2C CTL clone and P815 tumor cells, as well as their stable transfectant clones of PD-L1 (P815/ PD-L1 nos. 1-3). (C) $2 \mathrm{C}$ cells were cultured for 4 hours with $51 \mathrm{Cr}$ labeled P815 (0) or three separate

P815/PD-L1 clones in the absence or presence $(\Delta \square \diamond)$ of $10 \mu \mathrm{g} /$ $\mathrm{ml}$ rat anti-PD-L1 mAb F(ab')2 at various effector-to-target (E/T) ratios, and the specific $51 \mathrm{Cr}$ release was measured. The averages and standard deviations of triplicate cultures are shown [4].
A
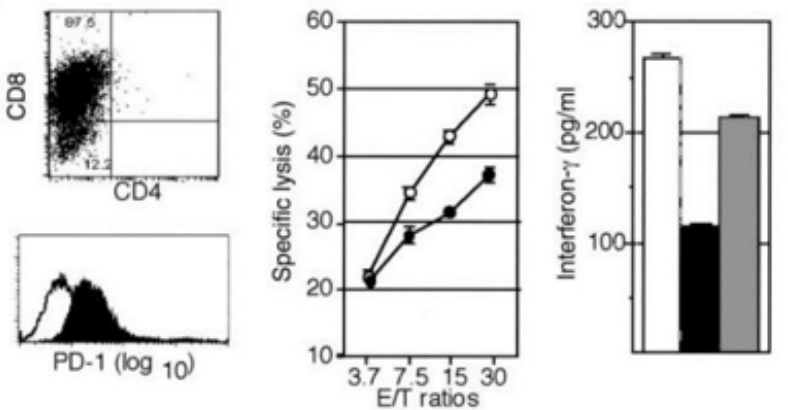

B
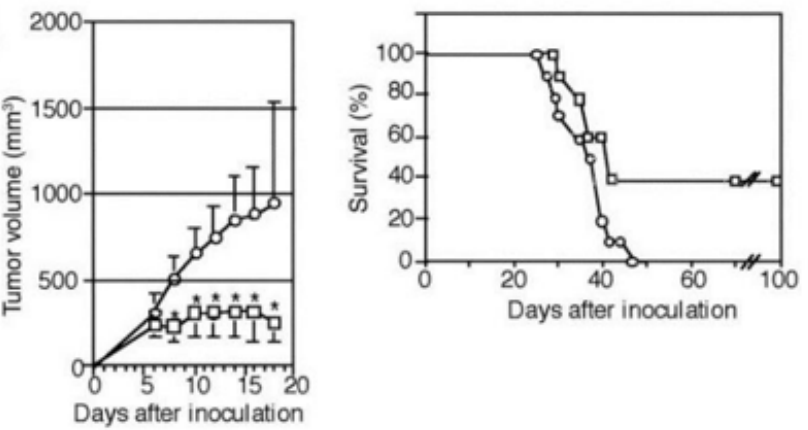

Figure 2: Injection of anti-PD-L1 mAb inhibits carcinogenesis in P815/PD-L1 cells in vivo. (To the left) In the DBA/2 mic, syngeneic P815 tumor-specific T lymphocytes were produced. (Center) The specific cytotoxicity of CD8+ T cells treated with 51Cr-labeled P815 (O) or P815/PD-L1 (F) cells at various effector-to-target ratios for 4 hours was evaluated. The average and standard deviations of triplicated cultures are shown. (Right) CD8+ T cells (2 x 106) were cocultured with 5 x 106 P815 (open bar) or P815/ PD-L1 cells for 24 hours in the absence (solid bar) or presence (shaded bar) of anti-PD-L1 mAb F(ab')2 (10 $\mu \mathrm{g} / \mathrm{ml})$, and IFN- $\gamma$ in the culture supernatants was measured using an enzymelinked immunosorbent assay. The average and standard deviations of triplicated cultures are shown. (B) DBA/2 mice (10 per group) were infected s.c. with 3 x 106 P815/PD-L1 cells, and then injected with $0.1 \mathrm{mg}$ of normal rat $\operatorname{lgG}(0)$ or anti-PD-L1 mAb (口) on days $1,3,5$, and 7 . The mean tumor sizes and SE of 10 mice are shown on the left, as well as their survival rates on the right. Student's t test yielded a $\mathrm{P}<0.01$ result [4]. 


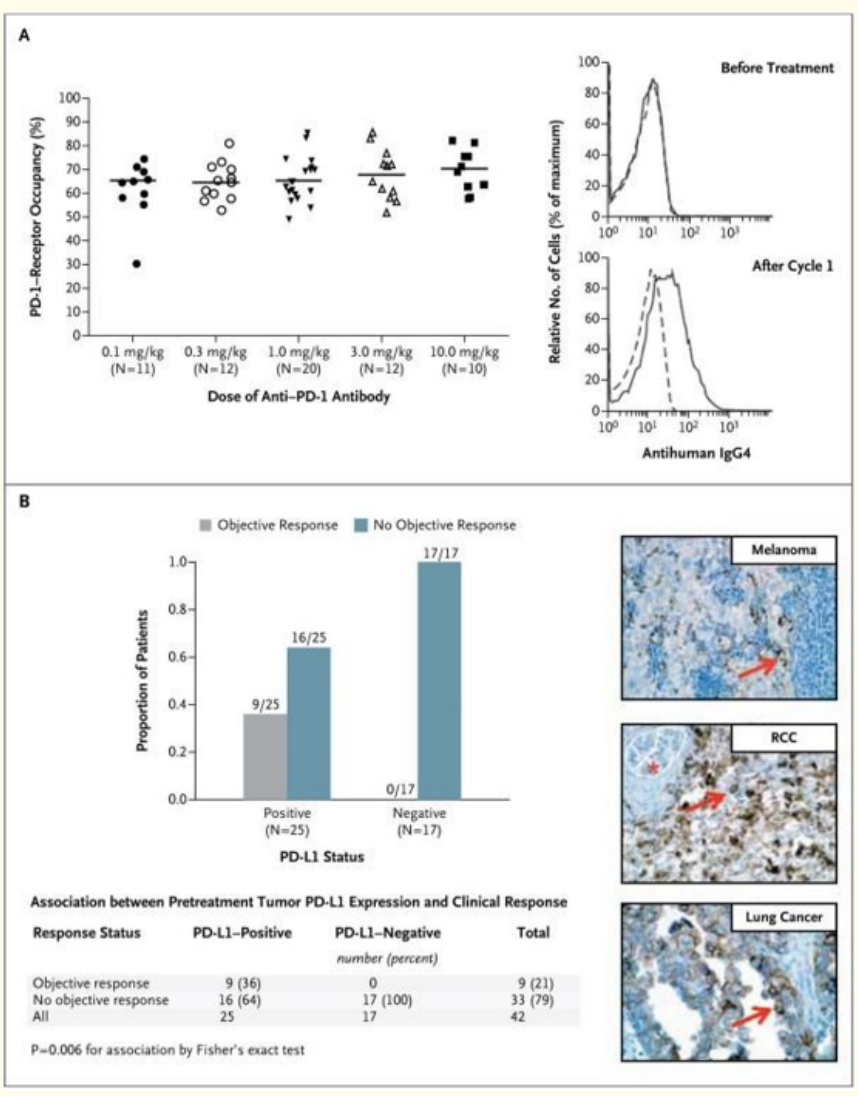

Figure 3: Panel A demonstrates PD-1-receptor occupancy by anti-PD-1 antibody. Pharmacodynamic and Molecular-Marker Assessments After one cycle ( 8 weeks) of therapy at a dosage of 0.1 to $10.0 \mathrm{mg}$ per kilogram every 2 weeks, PD-1-receptor occupancy on circulating $\mathrm{T}$ cells in 65 patients with melanoma is shown on the left graph. Median values are denoted by bars. The graphs on the right show PD-1 receptor occupancy on CD3-gated peripheral blood mononuclear cells from a patient with melanoma who got $0.1 \mathrm{mg}$ per kilogram before and after one treatment cycle from a patient with melanoma who received $0.1 \mathrm{mg}$ per kilogram (bottom). To identify injected anti-PD-1 antibody coupled to PD-1 molecules on the cell surface, cells were labeled with biotinylated antihuman IgG4. Streptavidin-phycoerythrin was used to detect the cells, which was followed by flow cytometric analysis. Isotype staining controls are indicated by dashed lines, while antihuman IgG4 is indicated by solid lines. Panel B illustrates the relation- ship between pretreatment tumor cell-surface expression of PD-1 ligand (PD-L1) and an objective response to PD-1 inhibition in 42 patients with advanced malignancies, as assessed by immunohistochemistry examination of formalin-fixed, paraffin-embedded specimens: 18 had melanoma, 10 had non-small-cell lung cancer,

7 had colorectal cancer, 5 had renal-cell carcinoma, and 2 had castration-resistant prostate cancer. The expression of PD-L1 on the surface of tumor cells was found to be strongly linked to a positive clinical outcome (graph at the left). There were no objective responses in patients with PD-L1-negative tumors. Two of the 25 patients with PD-L1-positive tumors who had not had a response at the time of data analysis are currently being evaluated. In cancer patients, a team of researchers from the University of California, San Diego, discovered a novel type of anti-PD-L1 monoclonal antibody. It was identified in melanoma, renal-cell cancer, lung adenocarcinoma and kidney failure patients' kidneys, lymph nodes and brain metastasies. Each specimen's arrow points to one of several tumor cells stained for PD-L1 on the surface membrane. The asterisk in the nephrectomy specimen shows a normal glomerulus that was PD-L1 negative [15].
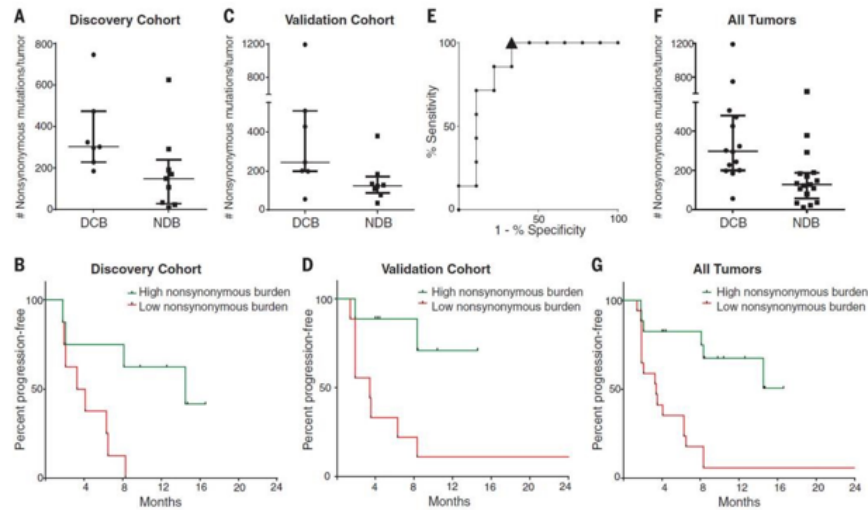

Figure 4: Nonsynonymous mutation load is linked to anti-PD-1 therapy's therapeutic benefit. (A) The prevalence of nonsynonymous mutations in tumors from DCB $(n=7)$ and NDB $(n=9)$ patients (median 302 versus 148, Mann-Whitney $\mathrm{P}=0.02$ ). (B) PFS 
The Role of Pd-L1 on Tumor Cells in Host Immune System Escape and Tumor Immunotherapy by Pd-L1 Blockade; Immune Checkpoint Blockade in Cancer Immunotherapy

in patients in the discovery cohort with malignancies with greater nonsynonymous mutation load $(\mathrm{n}=8)$ compared to tumors with

lower nonsynonymous mutation burden $(\mathrm{n}=8)$ (HR 0.19, 95 percent CI 0.05 to 0.70 , log-rank $P=0.01$ ). (C) Nonsynonymous mutation load in DCB-positive cancers $(n=7)$ versus NDB-positive tumors $(n=8)$ in the validation cohort (median 244 versus 125 , Mann-Whitney $P=0.04$ ). (D) PFS in patients in the validation cohort with tumors with higher nonsynonymous mutation load (n $=9)$ vs tumors with lower nonsynonymous mutation burden $(n=$ 9) (HR

$0.15,95$ percent $C I 0.04$ to 0.59 , log-rank $\mathrm{P}=0.006$ ). (E) In the discovery cohort, the ROC curve shows the association of nonsynonymous mutation load with DCB. The AUC is 0.86 (95 percent confidence interval: 0.66-1.05. Null hypothesis test: $\mathrm{P}=0.02$ ). The triangle represents the cut-off for 178 nonsynonymous mutations.

(F) For the complete set of sequenced tumors, nonsynonymous mutation load in patients with DCB ( $n=14)$ over those with NDB ( $\mathrm{n}=17$ ) (median 299 versus 127, Mann-Whitney $\mathrm{P}=0.0008$ ). (G) PFS in the whole set of sequenced tumors in those with higher nonsynonymous mutation load $(\mathrm{n}=17)$ compared to those with lower nonsynonymous mutation burden ( $\mathrm{n}=17$ ) (HR 0.19, 95 percent CI 0.08-0.47, log-rank $\mathrm{P}=0.0004$ ). The median and interquartile ranges of total nonsynonymous mutations are given in $(A),(C)$, and $(F)$, with individual values for each tumor represented by dots [19].
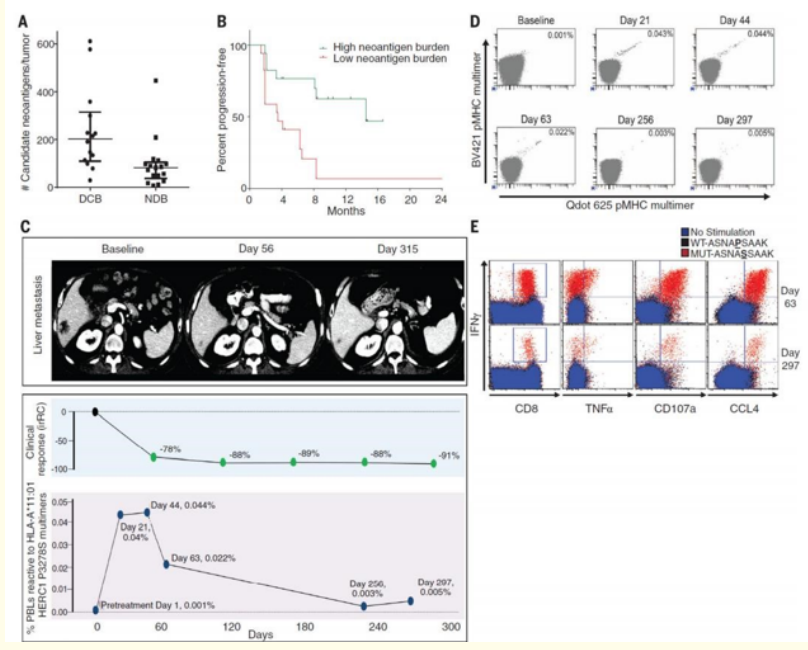

Figure 5: Neoantigen load in patients with DCB $(n=14)$ versus

NDB ( $n=17)$ throughout the whole collection of sequenced tumors (median 203 versus 83, Mann-Whitney $\mathrm{P}=0.001$ ). PFS in cancers with a greater candidate neoantigen load $(n=17)$ vs. tumors with a lower candidate neoantigen burden $(n=17)$ (log-rank $\mathrm{P}=0.002$, HR 0.23, 95 percent CI 0.09 to 0.58). (C) (Top) Pre- and post-treatment computed tomography (CT) scans of a liver metastasis. Change in radiographic reaction in the middle. In peripheral blood, the magnitude of the HERC1 P3278S reactive CD8+ T cell response was assessed. (D)Before and after pembrolizumab therapy, the percentage of CD8+ T cells in serially obtained autologous PBLs that recognize the HERC1 P3278S neoantigen (ASNASSAAK). Each neoantigen is represented by the events in the double positive position indicated with black dots, which are a unique combination of two fluorescently labeled peptide-MHC complexes(each axis is displayed separately). Neoantigen-specific T cells are represented by the events in the double positive position indicated with black dots. The amount of CD8+ MHC multimer+ cells is indicated by the percentages in Figure 4. Neoantigen load in patients with DCB $(n=14)$ versus NDB $(n=17)$ throughout the whole collection of sequenced tumors (median 203 versus 83, Mann-Whitney $\mathrm{P}=$ 0.001). (B) PFS in tumors with a high candidate neoantigen load $(\mathrm{n}=17)$ vs. tumors with a low candidate neoantigen burden $(\mathrm{n}=$ 17). (log-rank $\mathrm{P}=0.002$, HR 0.23, 95 percent CI 0.09 to 0.58). (C) (Top) Before and after therapy, representative computed tomography (CT) pictures of a liver metastasis. Change in radiographic response (middle). In peripheral blood, the magnitude of the HERC1 P3278S reactive CD8+ T cell response was assessed. (D) Before and after pembrolizumab therapy, the percentage of CD8+ T cells in serially collected autologous PBLs that recognize the HERC1 P3278S neoantigen (ASNASSAAK). Each neoantigen is represented by two fluorescently labeled peptideMHC complexes (shown individually on each axis); neoantigen-specific T cells are represented by events in the double positive position marked with black dots. The number of CD8+ MHC multimer+ cells is shown as a percentage [19]. 


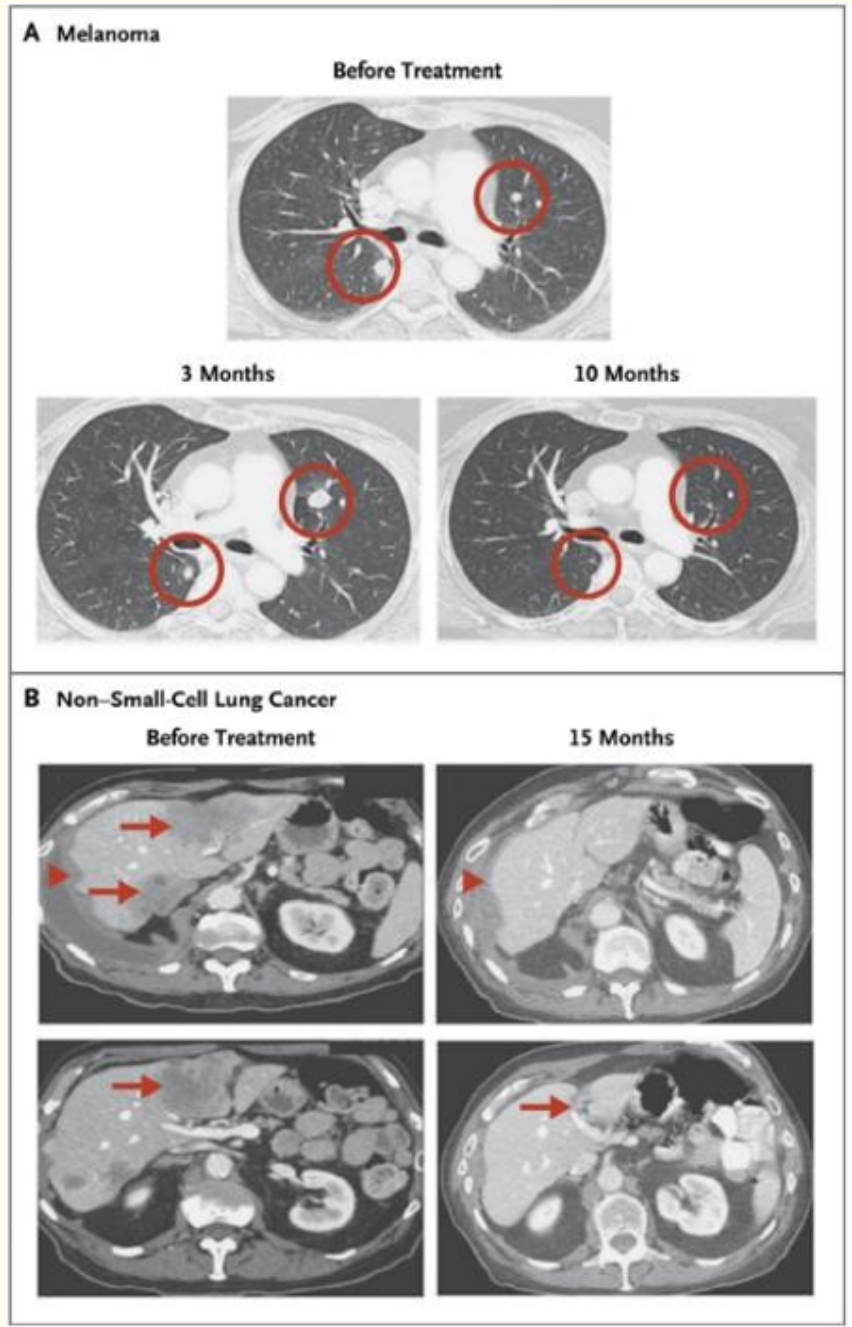

Figure 6: A full response was shown on computed tomography after receiving $3 \mathrm{mg}$ of anti-PD-L1 antibody per kilogram in a patient with melanoma who got $3 \mathrm{mg}$ of anti-PD-L1 antibody per kilogram. At 6 weeks and 3 months, circles show an initial increase in the size of lung nodules, followed by full regression at 10 months (i.e., an immune-related pattern of response). Panel $B$ shows a partial response in the liver (arrows) and right lung pleura (arrowheads) 15 months after receiving $10 \mathrm{mg}$ of antiPD-L1 antibody per kilogram in a patient with non-smallcell lung cancer (nonsquamous subtype) who got $10 \mathrm{mg}$ of anti-PD-L1 antibody per kilogram [18].

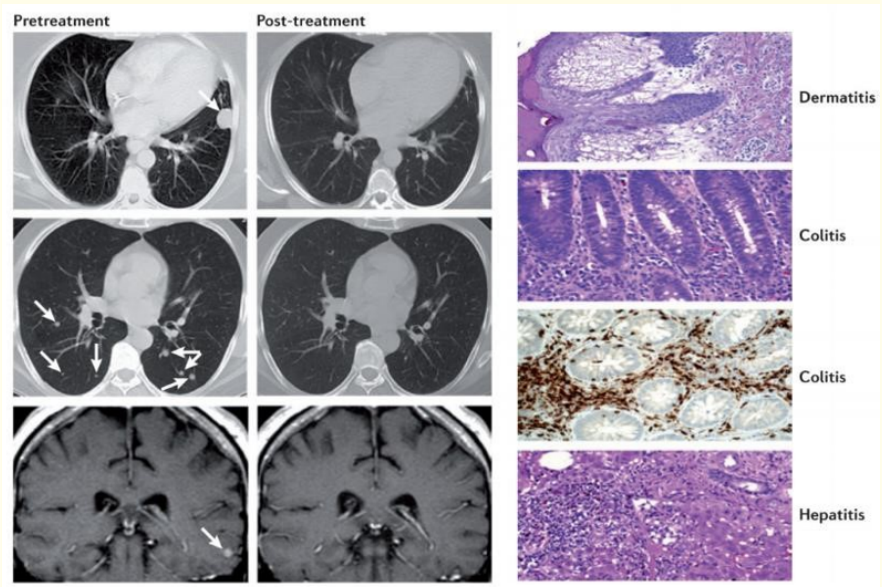

Figure 7: Antibody blockage of the CTLA4-mediated immune checkpoint causes clinical responses and immunological-mediated toxicity. Examples of lung (top two panels) and brain (lower panel) metastases in a melanoma patient treated with the CTLA4 antibody, ipilimumab, are shown on the left of the figure. Immunerelated 'on-target' toxicities can occur in $25-30 \%$ of patients receiving prolonged anti-CTLA4 treatment. However, with the short period utilized in the Phase III trial that resulted to the approval of ipilimumab, the prevalence of severe adverse effects was reduced (10-15 percent). The US Food and Drug Administration (FDA) recommended this short-course treatment ( 4 doses at a cost of US $\$ 30,000$ each dosage). The skin (dermatitis) and the colon are two common tissues impacted by immune-related toxicities from anti-CTLA4 medication, as indicated on the right of the picture (colitis). Tissues that do not regenerate as quickly as the skin and colon, such as the lung and liver, as well as the pituitary and thyroid glands, are less likely to be impacted. Anti-CTLA4 therapy's immune side effects are generally adequately controlled by systemic steroids and, where systemic steroids are ineffective, tumour necrosis factor (TNF) blockers. Even after a treatment of steroids, ongoing tumor responses are common. (2003) National Academy of Sciences, USA is used with permission to reprint this figure [9]. 

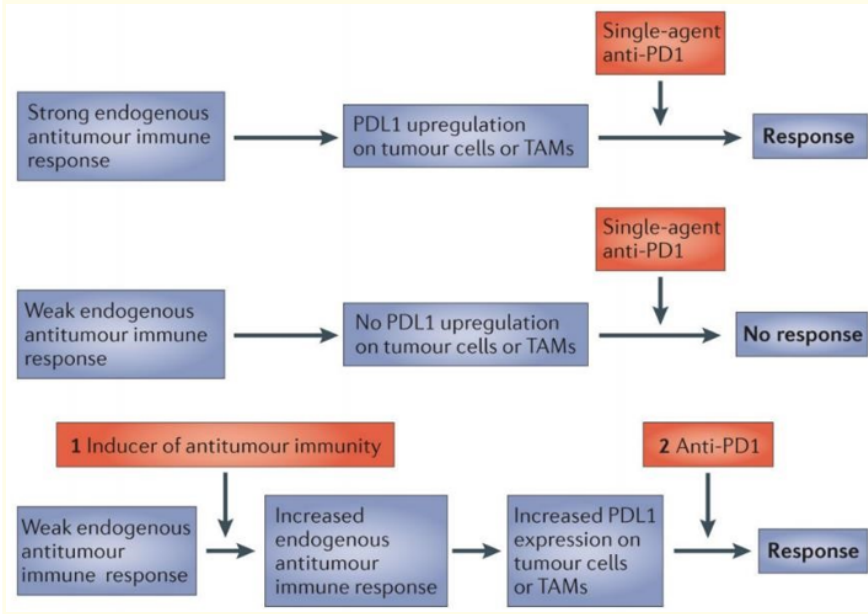

Figure 8: The adaptive immune resistance mechanism and its implications for cancer treatment. According to the adaptive immune resistance mechanism, blocking an induced immunecheckpoint protein, such as programmed cell death protein 1 (PD1), as a single intervention will only cause tumor regressions if there is a pre-existing antitumour immune response that can be 'unleashed' when the pathway is blocked. Tumors respond by upregulating immune-checkpoint ligands, thus multiple treatments, such as vaccinations, that generate a de novo antitumour immune response may not result in tumor regression. As a result, combining the two treatments might result in tumor regression in individuals who would not have responded to either treatment alone. PDL1 stands for PD1 ligand 1; TAM stands for tumor-associated macrophage [9].

Future prospects: biomarkers and combinations

FDA approval of anti-CTLA4 therapy and reports of encouraging clinical data for antiPD1 therapy has engendered a new-found awareness among oncologists of the potential antitumor activity of a patient's immune system. These immune checkpoints are a tiny fraction of the receptors and ligands that have been defined by genetic and biological analyses to inhibit specific types of immune responses at various levels. It is possible that specific oncogenic pathways, such as PI3K AKT or STAT3, may induce the expression of specific immunoinhibitory molecules. Anti-CTLA4 therapy strong- ly enhances the amplitude of vaccine-induced antitumor responses in many poorly immunogenic tumour models. These therapies may therefore force the tumors to upregulate immune checkpoints that can be blocked.

\section{Bibliography}

1. Burnet M. "Cancer-A Biological Approach I. The Processes Of Control”. British Medical Journal 1 (1957): 779-786.

2. Chambers C A., et al. "CTLA-4-mediated inhibition in regulation of $\mathrm{T}$ cell responses: Mechanisms and manipulation in tumor immunotherapy". Annual Review of Immunology 19 (2001): 565-594.

3. Gianchecchi E., et al. "Recent insights into the role of the PD-1/ PDL1 pathway in immunological tolerance and autoimmunity". Autoimmune Review 12 (2013): 1091-1100.

4. Iwai Y., et al. "Involvement of PD-L1 on tumor cells in the escape from host immune system and tumor immunotherapy by PD-L1 blockade". Proceedings of the National Academy of Sciences of the United States of America 99 (2002): 12293-12297.

5. Leach D R., et al. "Enhancement of antitumor immunity by CTLA4 blockade”. Science (80-. ). 271 (1996): 1734-1736.

6. Iwai Y., et al. "Cancer immunotherapies targeting the PD-1 signaling pathway". Journal of Biomedical Science 24 (2017): $1-11$.

7. Ciardiello F., et al. Annals of Oncology Advance Access. Annals of Oncology (2016): 1055-1061.

8. Lei Q., et al. "Resistance Mechanisms of Anti-PD1/PDL1 Therapy in Solid Tumors". Frontiers in Cell and Developmental Biology 8 (2020): 672.

9. Pardoll D M. "The blockade of immune checkpoints in cancer immunotherapy". Nature Review on Cancer 12 (2012): 252264.

10. Dammeijer F., et al. "The PD-1/PD-L1-Checkpoint Restrains T cell Immunity in TumorDraining Lymph Nodes". Cancer Cell 38 (2020): 685-700.e8.

11. Kuol N., et al. "Apostolopoulos, V. PD-1 / PD-L1 in disease". 10 (2018): 149-160.

12. Zhou Y., et al. "PD-1 and PD-L1 expression in 132 recurrent 
The Role of Pd-L1 on Tumor Cells in Host Immune System Escape and Tumor Immunotherapy by Pd-L1 Blockade; Immune Checkpoint Blockade in Cancer Immunotherapy

nasopharyngeal carcinoma: The correlation with anemia and outcomes". Oncotarget 8 (2017): 51210-51223.

13. Han Y., et al. "PD-1/PD-L1 pathway: current researches in cancer”. American Journal of Cancer Research 10 (2020): 727-742.

14. Wu X., et al. "Application of PD-1 Blockade in Cancer Immunotherapy". Computational and Structural Biotechnology Journal 17 (2019): 661-674.

15. Daskivich T J and Belldegrun A. "Safety, activity, and immune correlates of anti-PD-1 antibody in cancer". European Urology 67 (2015): 816-817.

16. Xue Y., et al. "Platinum-based chemotherapy in combination with PD-1/PD-L1 inhibitors: preclinical and clinical studies and mechanism of action". Expert Opinion on Drug Delivery 18 (2021): 187-203.

17. Fransen M F., et al. "Tumor-draining lymph nodes are pivotal in PD-1/PD-L1 checkpoint therapy". JCI Insight 3 (2018): 1-6.

18. Brahmer J R., et al. "Safety and Activity of Anti-PD-L1 Antibody in Patients with Advanced Cancer". The New England Journal of Medicine 366 (2012): 2455-2465.

19. Rizvi N A., et al. HHS Public Access 348 (2016): 124-128.

20. Massard C., et al. "Safety and efficacy of durvalumab (MEDI4736), an anti-programmed cell death ligand-1 immune checkpoint inhibitor, in patients with advanced urothelial bladder cancer". Journal of Clinical Oncology 34 (2016): 31193125.

21. Ishida Y., et al. "Induced expression of PD-1, a novel member of the immunoglobulin gene superfamily, upon programmed cell death". EMBO Journal 11 (1992): 3887-3895.

22. Xiao Q., et al. "Biological drug and drug delivery-mediated immunotherapy". Acta Pharmaceutica Sinica B 11 (2021): 941960.

23. Chen X., et al. "Epigenetic strategies synergize with PD-L1/ PD-1 targeted cancer immunotherapies to enhance antitumor responses". Acta Pharmaceutica Sinica B 10 (2020): 723-733.

24. Chen R., et al. "Phase II study of the efficacy and safety of pembrolizumab for relapsed/refractory classic Hodgkin Lymphoma”. Journal of Clinical Oncology 35 (2017): 2125-2132.
25. Ruf M., et al. "PD-L1 expression is regulated by hypoxia inducible factor in clear cell renal cell carcinoma". International Journal of Cancer 139 (2016): 396-403.

26. Shao J W., et al. "In vitro and in vivo anticancer activity evaluation of ursolic acid derivatives". European Journal of Medicinal Chemistry 46 (2011): 2652-2661.

Volume 4 Issue 10 October 2021 (C) All rights are reserved by Mehmet Eraslan. 